\title{
Energy
}

\section{DEVELOPMENT AND PILOT TESTING OF MODULAR DYNAMIC \\ THERMOMECHANICAL PULP MILL MODEL TO DEVELOP ENERGY REDUCTION STRATEGIES}

Final Report

By

D. W. Coffin

October 1996

Work Performed Under Contract No. FG07-90CE40937

For

U.S. Department of Energy

Assistant Secretary for Energy Efficiency and Renewable Energy Washington, DC

By

The Institute of Paper Science and Technology

Atlanta, Georgla 


$$
\begin{aligned}
& \text { RECE } 1, \mathrm{Fm}^{\circ} \\
& \text { DEC } 1 \text { i } . \\
& \mathrm{OS}_{1} .
\end{aligned}
$$

\section{DEVELOPMENT AND PLOT TESTING OF MODULAR DYNAMIC THERMOMECHANICAL PULP MUL MODEL TO DEVELOP ENERGY REDUCTION STRATEGIES}

Final Report

D. W. Coffin

October 1996

Work Performed Under Contract No. FG07-90CE40937

For

U.S. Department of Energy Assistant Secretary for Energy Efficiency and Renewable Energy Washington, DC

By

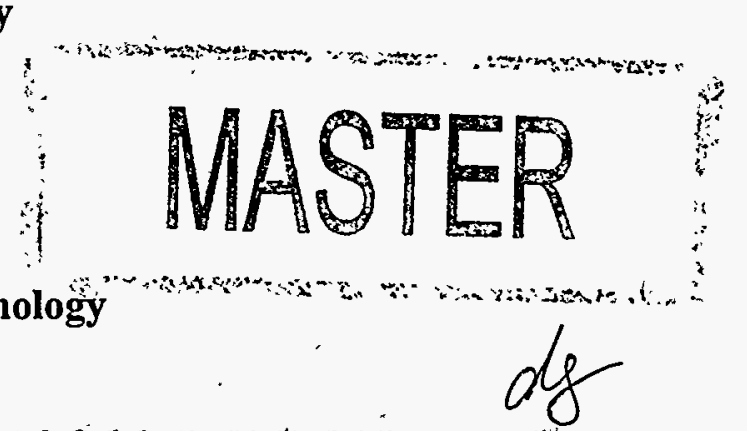




\section{DISCLAIMER}

Portions of this document may be illegible in electronic image products. Images are produced from the best available original document. 
DEVELOPMENT AND PILOT TESTING OF MODULAR DYNAMIC THERMOMECHANICAL PULP MLL MODEL TO DEVELOP ENERGY REDUCTION STRATEGIES

Contract Number: DE-FG07-90CE40937

Final Report to

The Office of Industrial Technology of the United States Department of Energy

Washington, D. C.

by

Douglas W. Coffin

The Institute of Paper Science and Technology

Atlanta, GA 


\begin{abstract}
With the development of on-line and real-time process simulations, one obtains the ability to predict and control the process; thus, the opportunity exists to improve energy efficiency, decrease material wastes, and maintain product quality. Developing the capability described in the previous sentence was the objective of the research program described in the following. A thermomechanical pulp mill was simulated using both a first principles model and a neural network. The models made use of actual process data and a model that calculated the mass and energy balance of the mill was successfully implemented and run at the mill on an hourly basis. The attempt to develop a model that accurately predicted the quality of the pulp was not successful. It was concluded that the key for a successful implementation of a real-time control model, such as a neural net model, is availability of on-line sensors that sufficiently characterize the pulp.
\end{abstract}




\section{TABLE OF CONTENTS}

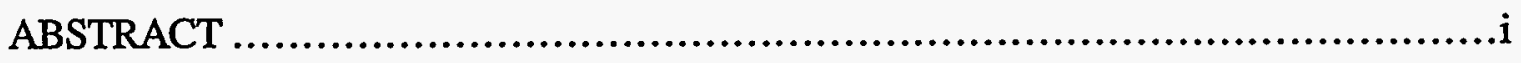

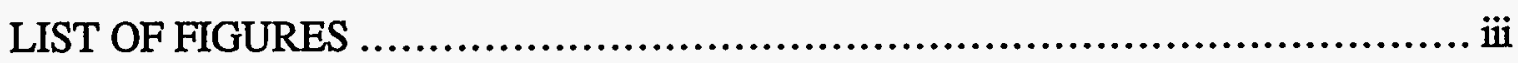

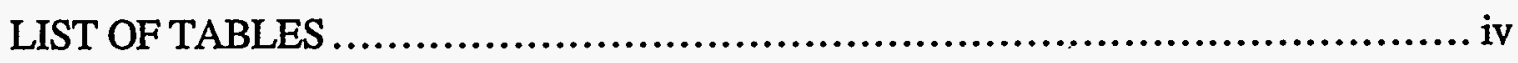

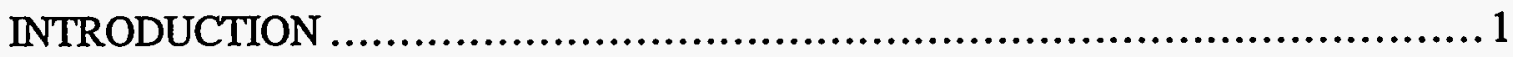

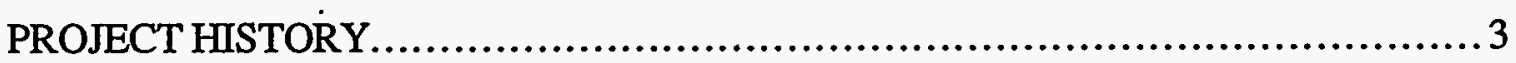

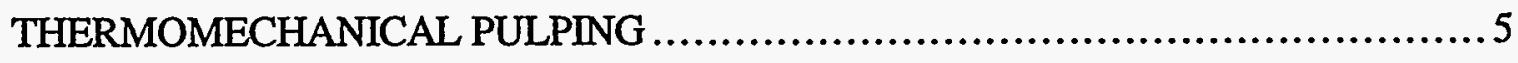

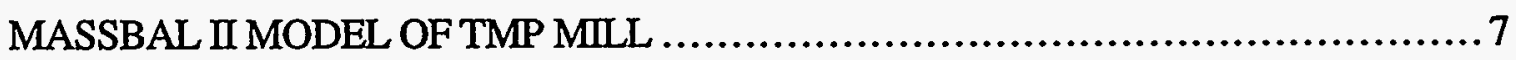

Model Components .......................................................

Model Assessment.................................................8

MASSBAL-PI Interface .................................................. 9

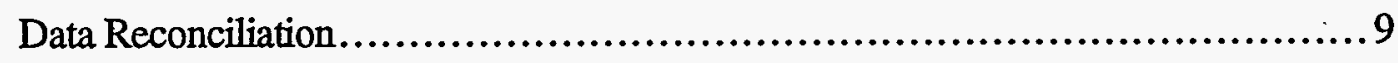

Implementation of MASSBAL II Model at Bowater ............................12

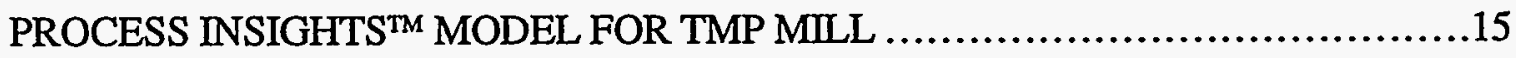

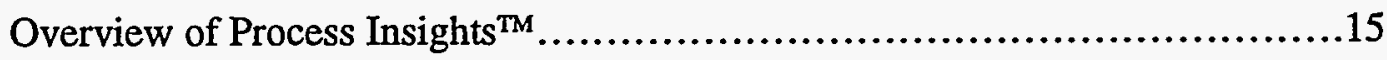

Model Variables ..........................................................16

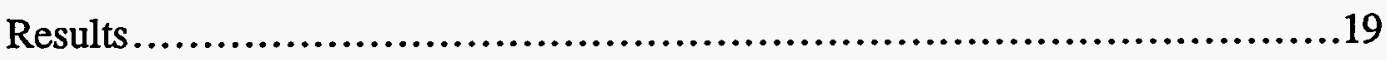

Analysis of Results............................................................

CONCLUSIONS AND RECOMMENDATIONS .................................24

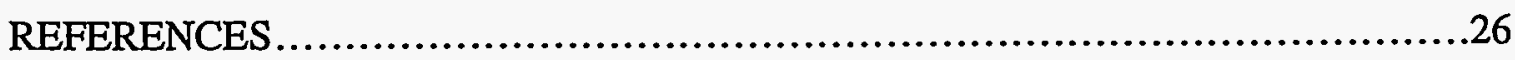

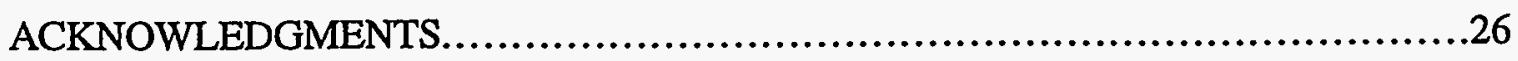




\section{LIST OF FIGURES}

Figure 1. Process Diagram of TMP Mill..............................................5

Figure 2. Simple Flow Diagram for Reconciliation ...............................10

Figure 3. Complex Flow Diagram for Reconciliation................................12

Figure 4. Example of the Graphical Output for MASSBAL Results......................13

Figure 5. Examples of Multiple Graphical Output of MASSBAL Results................14 


\section{LIST OF TABLES}

Table 1: MASSBAL Equipment Modules Used in TMP Model.

Table 2: MASSBAL Parameters for Refiners. 8

Table 3. Balances for Simple Flow Model. .11

Table 4. Balances for Complex Flow Model.

Table 5. Input Parameters for Process Insights ${ }^{\mathrm{TM}}$. Model.

Table 6. Output Parameters for Process Insights ${ }^{\mathrm{TM}}$ Model

Table 7. Additional Input Parameters Compiled by Bowater. .18

Table 8. Summary of Statistics for Process Insights ${ }^{\mathrm{TM}}$ Variables.

Table 9. Cross Correlation Coefficients for Input Parameters. .21

Table 10. Cross Correlation Coefficients for Output Parameters .21

Table 11. Cross Correlation Coefficients for Input versus Output Parameters .22

Table 12. Results from Linear Regression 23 


\section{INTRODUCTION}

Process simulations are utilized, often, in the design, operation, and analysis of a process. A simulation that makes use of actual process data and provides rapid feedback can increase the efficiency of the process and be used for open-loop control. A simulator that provides real-time predictions could potentially be used for closed loop control. With the ability to predict the quality of a product based on the process parameters in real-time, a process can be optimized; thus, reducing energy consumption, operating costs, and material usage while maintaining acceptable quality of the product.

Process operators and their managers usually can not make immediate use of off-line models of their process. These are the people that are striving to optimize the process while maintaining a product of acceptable quality. They need to make hourly and daily decisions and can not wait for the results of an off-line model. Often, the decisions made by the operators require more process data then is available, and they rely on personal experience, intuition, and trial and error. If the process information could be obtained from an on-line simulation program that links material and energy flows with product quality, informed and rational decisions about process changes could be made. In addition, the process simulator could be used to optimize and control the process.

The development of just such a process simulator was the objective of a research project conducted by the Institute of Paper Science and Technology (IPST) in Atlanta, GA and sponsored by the Office of Industrial Technology (OIT) of the US Department of Energy (DOE). The objectives of this project were to develop a real-time process simulation model and demonstrate its use as a means of process analysis and optimization. The focus of this work was to develop a model of the thermomechanical pulp (TMP) mill at the Bowater Incorporated mill in Catawba, SC.

The thrust of this work was to develop a new process simulation software that combined the performance attributes feature of IPST's MAPPS (Modular Analysis for Pulp and Paper Systems) program with the MASSBAL program of SACDA Incorporated of London, Ontario. The new program would have been capable of dynamic simulation and process optimization while predicting and maintaining product quality. The effort to develop this package was not successful, and separate MASSBAL and MAPPS models were developed. 
In an attempt to meet the original objectives of the program, a process simulation tool based on neural network theory and fuzzy logic was utilized. Process Insights ${ }^{\mathrm{TM}}$ of Pavilion Technologies, Austin TX, was used to model the refiner at the Bowater, Catawba mill. The neural network model trains a process model based on the historical process data. The historical data must adequately capture the process such that the desired predicted output can indeed be represented by the chosen input parameters. The initial attempt to model the process at the Bowater mill led to the conclusion that the input data did not adequately represent the process. To obtain the necessary historical data would have required more resources then were available. Therefore, it was decided to shut the project down and summarize the results that had been obtained to date.

The decision to conclude the project before obtaining the objectives was arrived at by looking at the entire history of the project, and the requirements to complete the project. Although the original objective of the project is technically sound and probably achievable, it was not achieved in this project for many other reasons. Much of the effort to combine MAPPS and MASSBAL was conducted by SACDA who experienced personnel changes during the development process with adverse effects on continuity of development and record keeping of the progress. In addition, the Principal Investigators at IPST changed several times throughout the project, and there was a loss of continuity. At present, process simulation is not a research focus at IPST, and development of MAPPS has been stopped. During the project, Bowater experienced a down sizing and became very limited in their ability to provide support for the project at the mill. Because of the failure of the combined MASSBAL-MAPPS program and the lack of progress, the enthusiasm level of Bowater personnel was low. To continue with the project would have required a large effort for data collection with the possibility of once again not being able to adequately model the process. Therefore, instead of trying to add new life to the project, it appeared more prudent to shut it down.

In the following, the results that were achieved in the project are reviewed. One development effort was implemented at the Mill. This was the MASSBAL model of the TMP process. A MASSBAL model for the mass and energy flow of the TMP mill was developed by SACDA, installed at the mill, run hourly, and utilized to develop a daily report. The exceptions report can be used to help identify possible problems at the TMP mill. 


\section{PROJECT HISTORY}

The research project reported on herein was started September 30, 1990 with a grant from the Department of Energy and continued through April 30, 1996. The recipient of this award was the Institute of Paper Science and Technology. A subcontract was awarded to SACDA Inc. to assist in the development of new software.

This project was being conducted as part of a larger project coordinated by the International Energy Agency (IEA) on control and modeling. This project, referred to as Annex VII, consisted of the following three projects: a project addressing on-line process monitoring conducted by PAPRICAN in Canada; the TMP modeling project conducted by IPST in the USA, and a project investigating methods for dealing with missing process data conducted by STFI in Sweden.

Work on the TMP modeling project began in April of 1991, and a kick-off meeting between IPST and SACDA was held April 23 and 24 in 1991. Shortly after this time, Bowater Incorporated agreed to participate in the project and host the implementation of the project at the TMP mill in Catawba, South Carolina. The original objectives and research plan for this project are given in the first project report of this program [1].

From 1991 through 1994, work focused on developing the new software that would incorporate the performance attributes feature of MAPPS [2] with MASSBAL [3]. Most of this work was conducted at SACDA, and a beta version of the new software apparently was developed. It appears that this beta version did not work. Most likely the MASSBALMAPPS combined model did not work because of the incompatibility of the two software architectures. The effort was deemed unsuccessful and work on developing the new software stopped. The development of this software was not recorded and it is unclear of exactly why this effort was unsuccessful. In an effort to obtain a model that would meet some of the original objectives of the project, a model of the TMP mill was made using SACDA's MASSBAL II program. The model was developed and a report of the model was prepared by SACDA in August of 1994 [4].

This MASSBAL model was installed at the Bowater Mill and interfaced to the Mill's Plant Information system. The MASSBAL model was updated and made operational at the mill. One of the changes was a modification to reflect the bypass of one of the screens at the 
mill. The model has the capability to reconcile data and identify process variables that do not fit well with the data. This model was used to develop an exceptions report, listing discrepancies between the model and the process data. The MASSBAL model was run every hour at the mill and a daily exceptions report was automatically compiled. This report can be used by process engineers and managers to identify possible problems in the process or faulty instrumentation. The model was also used to evaluate a "what-if" scenario of screw press capacity expansion for the refiner start-up de-bottlenecking.

The MASSBAL II'model of the Bowater TMP mill only predicts the energy and mass flow of the process. MASSBAL can not predict the quality of the pulp, which was the reason for the attempt to incorporate MAPPS into MASSBAL. In 1991 a MAPPS model of the TMP mill was developed at IPST [5]. Two models were developed, one was a Newsprint line and one was a lightweight coated line. These models were developed as a Master student's research project and the results of this study are presented in Reference [5]. The models were used to develop sensitivities of quality parameters to various process parameters, but little correlation was found. In 1995, the existing MAPPS model was investigated, and found to have a flaw in amount of heat loss for the refiner module. This change was made, but no further development of the MAPPS model was completed.

In late 1994, the decision was made to pursue a new process software package that would be able to predict quality output. Process Insights ${ }^{\mathrm{TM}}$ (Pavilion Technologies, Austin Texas) [6] uses neural network theory to train a model of the process based on historical process data. Therefore, quality data can be chosen as output variables to be predicted from input process data. This software was purchased in December of 1994 and installed on site at the Bowater Mill. In addition, IPST purchased and installed Process Insights ${ }^{\mathrm{TM}}$ at IPST. As the MASSBAL model was being completed, the Process Insights ${ }^{\mathrm{TM}}$ modeling was started. Initial process data and quality data were gathered at the mill and sent to Pavilion Technologies where a model was developed. Initial modeling showed that the data provided by Bowater was insufficient to develop a predictive model for the process. It was decided that a large effort to collect new process data would have to be carried out, and that this was not feasible. At this point, it was decided to stop the project. 


\section{THERMOMECHANICAL PULPING}

Thermomechanical pulping (TMP) is a process for producing papermaking fibers from wood chips by treating the fibers with both heat and mechanical action. Wood chips are steamed prior to and during the mechanical treatment or refining. The application of steam softens the chips and the refining separates the chips into fibers. The softening of the chips makes it easier to separate the fibers, and a TMP operation produces fibers of greater length and lower shives or fiber bundles then a regular refining process. After the fibers are separated, they are curled and twisted, and a sheet of low strength is obtained. Latency is used to describe the dormant properties of the pulp that can be re-gained through increased temperature, referred to as removal of latency. The fibers are soaked in hot water to remove this latency and produce a pulp of straight fibers. After this stage, the fibers are cleaned, and shives are removed with screens. At this point bleaching of the fibers would be completed and the pulp would be delivered to the paper machine.

A process diagram of a TMP line similar to that at the Bowater mill in Catawba is shown in Figure 1. At the Bowater TMP mill pulp is produced for both newsprint and lightweight coated paper. There are three lines for the lightweight coated stock, and four lines for the newsprint stock.

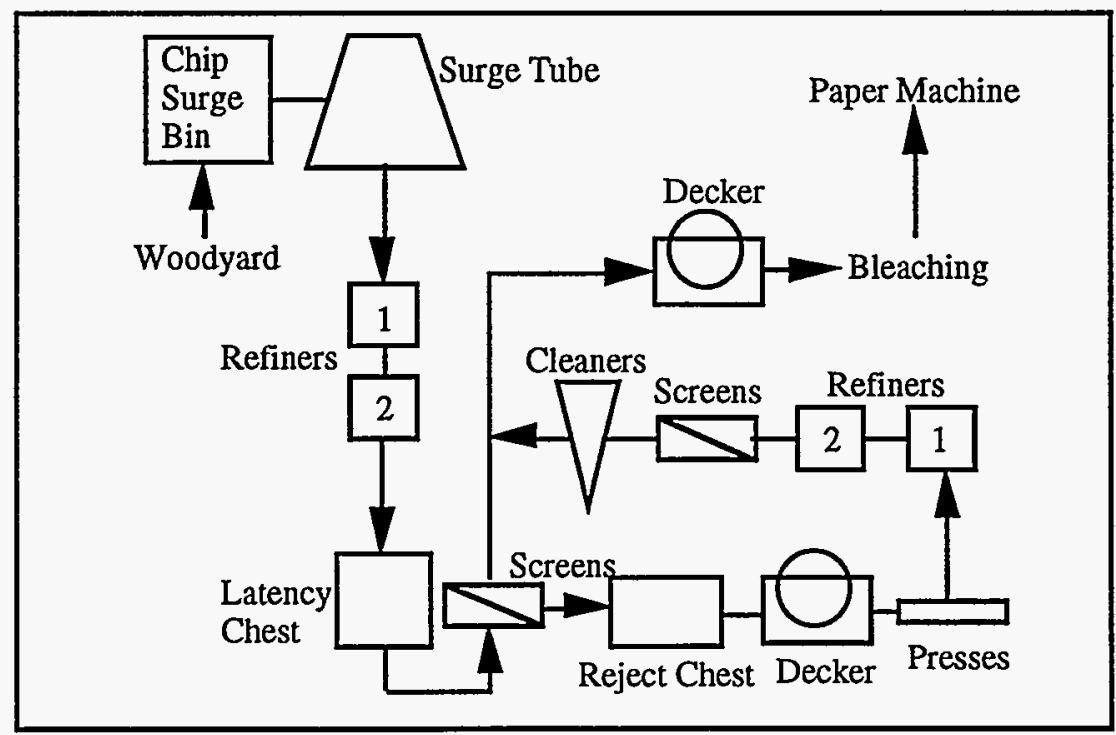

Figure 1. Process Diagram of TMP Mill.

The MASSBAL model, described in the next section, simulates the newsprint side of the TMP mill. Raw wood chips, from the wood yard, are feed into two chip surge bins where 
they are preheated with 60 psig steam. They are steamed in the chip surge tubes and then sent to four duplicate refiners. The lines consist of a surge tube, chip screw feeder, primary refiner and cyclone, and a secondary refiner and cyclone. At the surge tube ,150 psig steam is added to soften the chips. The chip screw feed regulates the flow of chips into the refiner and dilution water is added to cool the refiner plates. The primary refiners break the chips down to the fiber level. Heat is generated in the refining and the cyclone is used to remove the steam from the pulp. The pulp is further refined in a secondary refiner and again steam is removed in the secondary cyclone.

The fibers are then placed in the latency chest where heat is added to allow the fibers to straighten out. At this point, the pulp flows to the screen surge chest where it is combined with rejects from one of the papermachines and some white water. This pulp then flows to the screen headbox where is it diluted with white water and prepared for screens. A series or screens remove shives (unseparated fiber bundles) and other oversized matter. The accepted pulp flows to a screen accept chest.

The rejects are sent to a reject storage chest. The consistency of the rejects is raised by removing water in a decker and a press. The rejects are then refined in a primary and secondary refiner. This newly refined pulp is sent through a series of screens and cleaners, and the accepted stock is added back to the main line.

The accepted stock is sent to a decker to thicken up the stock and separate the clear and cloudy water. The stock is then sent to storage tanks followed by bleaching. The decker for accept flow is a boundary of the MASSBAL model.

The TMP process is very energy intensive process and most of the energy is expended in breaking up the primary fiber wall in the refiners. It is estimated that $2,400 \mathrm{kWh}$ are expended to produce each ton of TMP. In 1993, 3.38 million tons of TMP were produced in the U. S. Assuming an energy cost of $\$ 0.04 / \mathrm{kWh}$, a $2 \%$ reduction in energy usage would result in a yearly industry savings of $\$ 7$ million. The price of market TMP is in the range of $\$ 300$ to $\$ 500$ per ton. It costs $\$ 100$ per ton for energy consumption; and therefore, a large percentage of the cost goes to energy usage. It is essential that energy consumption in producing TMP be minimized. Of course, the energy savings can not be at the cost of pulp quality. 


\section{MASSBAL I MODEL OF TMP MILL}

\section{Model Components}

A MASSBAL II model of the TMP process described in the last section was developed by SACDA. MASSBAL II is a simulation software that is used to determine the steady-state heat and mass balances of a process. The model consists of modules representing components of the equipment and constraints to the process. This provides a system of equations that is solved simultaneously. The following discussion is taken from Reference [4], which outlined the MASSBAL model.

The following MASSBAL equipment modules were used in the TMP model: ADDER, DIVIDER, FLASH, GENUNIT, HEADER, REACTOR, SEPARATOR, SINK, and SOURCE. The plant equipment modeled with these modules is given Table 1.

Table 1: MASSBAL Equipment Modules Used in TMP Model.

\begin{tabular}{|l|l|}
\hline Module & Process Component \\
\hline ADDER & $\begin{array}{l}\text { Chip Bin A, Chip Bin B, Liquid Phase Separators, } \\
\text { Latency Chests, Screen Surge Chest, Screen Headbox, } \\
\text { Screen Accepts Chest, Screen Rejects Chest, Reject } \\
\text { Screen Accepts Chest, Reject Screen Rejects Chest, } \\
\text { Various Piping Connections }\end{array}$ \\
\hline DIVIDER & $\begin{array}{l}\text { 60 PSIG Steam Header, 150 PSIG Steam Header, } \\
\text { Blow Back Steam Headers, Reject Screen Headbox, } \\
\text { Various Piping Connections }\end{array}$ \\
\hline FLASH & Primary Cyclones, Secondary Cyclones \\
\hline GENUNIT & Mainline Deckers \\
\hline HEADER & Press Filtrate Chest \\
\hline REACTOR & $\begin{array}{l}\text { Primary Refiner, Secondary Refiner, Primary Reject } \\
\text { Refiner, Secondary Reject Refiner }\end{array}$ \\
\hline SINK & $\begin{array}{l}\text { Primary Screens, Reject Dewatering Presses, Reject } \\
\text { Screens, Primary Cleaners, Secondary Cleaners, } \\
\text { Monitor Cleaners, Tertiary Cleaners, RCC Mainline } \\
\text { Deckers }\end{array}$ \\
\hline SOURCE & $\begin{array}{l}\text { 45 PSIG Steam, 38 PSIG Steam, 26 PSIG Steam, RCC } \\
\text { Rejects, TMP HD Chest, Cloudy White Water Chest, } \\
\text { Clear White Water Chest }\end{array}$ \\
\hline $\begin{array}{l}\text { 60 PSIG Steam, 150 PSIG Steam, Chip Feed, Clear } \\
\text { White Water, Cloudy White Water, Bleach, No. 1 TMP } \\
\text { RCC, NO. 2 TMP RCC, NO. 2 PM Rejects, NO. A } \\
\text { Cloudy White Water }\end{array}$ \\
\hline
\end{tabular}

The main line TMP refiners and the reject refiners were all modeled as a group using three MASSBAL equipment modules. These modules were the ADDER, REACTOR, and GENUNIT. The ADDER is configured to combine the stock, plate and case dilution, 
bleach, and blow back steam at the entry to the refiners. The reactor module provides the extent of the reaction within the refiner. The GENUNIT is used to calculate the steam generated from the refiner's duty. Table 2 lists parameters defined as block variables for the refiners.

Table 2: MASSBAL Parameters for Refiners.

\begin{tabular}{|l|l|l|}
\hline Variable name & Description & Typical Value \\
\hline YIELD (\%) & $\begin{array}{l}\text { Percentage Yield from refining = (mass } \\
\text { flow of pulp out)/(mass flow of chips } \\
\text { in)*100 }\end{array}$ & $95 \%$ \\
\hline LOAD (MW) & Total refining energy applied to pulp & measured Value \\
\hline RENERG (\%) & $\begin{array}{l}\text { Percentage of applied energy that is } \\
\text { available for steam generation. }\end{array}$ & $65 \%$ \\
\hline $\begin{array}{l}\text { SPECIFIC ENERGY } \\
\text { (HPD/Ton) }\end{array}$ & Specific energy of refining & calculated value \\
\hline
\end{tabular}

The following equations are incorporated in to the refiner group to satisfy model constraints:

$$
\begin{aligned}
& \text { EXTENT(\%) }=100-\text { YIELD }(\%) \\
& \text { DUTY }(\mathrm{BTU} / \mathrm{Hr})=\text { LOAD }(\mathrm{MW}) * \text { RENERG }(\%) * \text { CONVERSION FACTOR } \\
& \text { SPECIFIC ENERGY }(\mathrm{HPD} / \mathrm{T})=\text { LOAD }(\mathrm{MW}) / \text { Tonnage } * \text { CONVERSION } \\
& \text { FACTOR }
\end{aligned}
$$

\section{Model Assessment}

The MASSBAL model tracks fiber, water, and dissolved solids through the TMP mill. No quality predictions are available with the current model. The model developed by Dodds [4] appeared to have been spliced together from two smaller models, and consequently not all recycle streams were connected. The dilution whitewater appears as several input headers, while the decker filtrate and screw press are represented as sewer streams. Likewise, low pressure steam generation and usage are not fully connected because the recompression units and boilers are off model. Modifications to the original model were made in order to reflect the current TMP process at Bowater. Several factors in the model are prescribed. For example, the $65 \%$ of the refiner shaft horsepower is added as enthalpy to the pulp outlet stream. These prescribed factors may not be correct, and could have a significant effect on the output. It was judged that the final model adequately 
simulated the Bowater TMP process, although some discrepancies exist. In addition, some convergence problems were encountered when large changes in the process occurred, such as shutting down or restarting one of the refining lines. Overall, the model gave a good representation of the process, and provided insights into the mass and energy flow of the process.

\section{MASSBAL-PI Interface}

The MASSBAL model was interfaced to the Plant Information System (PI) so that data could be read directly from the PI database, the model run, and the MASSBAL output be placed back on the PI database.

This was accomplished using the RUNCHANGE feature of MASSBAL This feature allows the model to be configured once, and multiple solutions obtained with different sets of operating constraints and parameters. The input parameters that are not fixed must be identified with the RUNCHANGE keyword, in a RUNCHANGE file. The interface program reads the RUNCHANGE file, retrieves the necessary data from the PI system and writes it o the RUNCHANGE file. The MASSBAL model is then run with the RUNCHANGE feature, and the solution is obtained using the latest PI data.

An output file from MASSBAL is then generated containing a list of PI database tags and a value for each tag. A second interface program then transfers the data to the PI database.

\section{Data Reconciliation}

Modern pulp and paper mills make thousands of different process and product measurements. These measurements are used as input to control the process and maintain the quality of the product. All the measurements contain error, and these errors are due to sources such as the ability to obtain a representative sample or the limitations of the sensors or lab equipment. In order to maintain product quality at a minimum cost, the threshold of these errors must be kept below some level. In order to maintain the process in control, one must be able to determine when the error in the reading becomes so large the value is meaningless. In other words, the accuracy of the process and quality output must be determined. 
The necessary measurement and testing accuracy can be maintained in a number of ways. An aggressive preventive maintenance program can be effective but expensive. Redundant sensors can provide the information necessary to evaluate the degree of error, but at large expense. A more powerful approach known as data reconciliation can be used to examine the internal consistency of the data in light of the physical laws such as mass and energy balance governing the process. This approach to this problem has been commonly used in the petrochemical industry and been found to be a very cost effective way to find measurement errors. Using data reconciliation software one can often get good results with only a $20 \%$ excess of measurement values over the minimum number needed to solve the balance. Published reports from Exxon have shown that failure to reconcile process data before analysis can lead to incorrect process adjustments and the associated financial losses [7]. Despite the obvious advantages of this methodology, it has been relatively little used in the pulp and paper industry, perhaps because of it's perceived mathematical complexity [8].

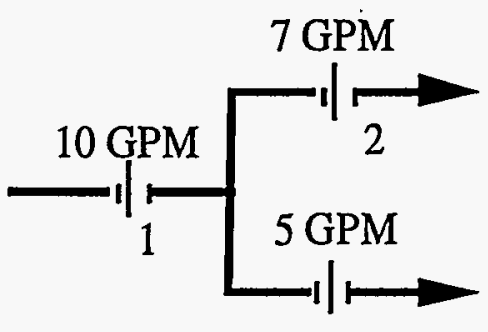

3

Figure 2. Simple Flow Diagram for Reconciliation

The following discussion will be used to describe data reconciliation. Figure 2 shows a simple pipe tee with three flow meters in the line. The mass balance requires that the incoming flow must equal the outgoing flow (flow $1=$ flow $2+$ flow3). This means that only two of the readings are independent, and the third value relies on the mass balance equation. Therefore, three balances can be written using each of the possible combinations of two of the data values. If all the balances had agreed, then the measurements would have been consistent. The measurements in Table 3 are not consistent and it is of interest to identify both measurement errors and the best balance to use for operating decisions. This is the problem of reconciling measurement data. 
Table 3. Balances for Simple Flow Model.

\begin{tabular}{|l|l|l|l|}
\hline & Flow 1 & Flow 2 & Flow 3 \\
\hline Measurement & 10 & 5 & 7 \\
\hline Balance One & 10 & 5 & 5 \\
\hline Balance Two & 10 & 3 & 7 \\
\hline Balance Three & 12 & 5 & 7 \\
\hline $\begin{array}{l}\text { "Best" Balance from } \\
\text { MASSBAL }\end{array}$ & 11.15 & 4.71 & 6.44 \\
\hline Error & +1.15 & -0.29 & -0.56 \\
\hline
\end{tabular}

There are an infinite number of measurement sets that would satisfy a mass balance, it is reasonable and standard practice to chose the balance which minimizes the sum of the squares of the error. This is a constrained optimization, since only parts of the variable space make physical sense. For example, solutions with negative flows and pressures may be mathematically valid but are not of engineering interest. In a slightly more formal sense, we can consider data reconciliation to be the process of transforming a set of measurements, which generally does not satisfy mass and energy balances to a new set which satisfies the balances.

As in regression analysis, the distribution of the data points about the "mean least squares balance" is a measure of the probability that an individual measurement is a valid member of that distribution, provided that we have enough excess data points (degrees of freedom) beyond what is required to define the balance. (If we have ten equations in the balance and fourteen data points, we have 4 degrees of freedom)

In the first example, there was not enough data to describe the quality of the individual measurements. However, if we add additional streams, as shown in Figure 3 and Table 4, we can begin to use the meters to cross-check one another. In such situations, we can say that measurements are more or less consistent with the rest of the data set. 


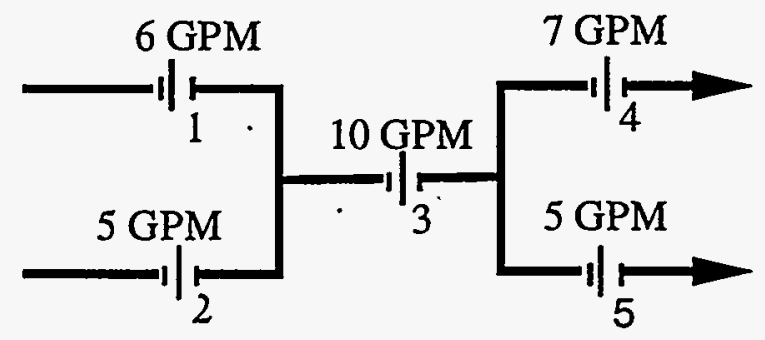

Figure 3. Complex Flow Diagram for Reconciliation

Table 4. Balances for Complex Flow Model.

\begin{tabular}{|l|l|l|l|l|l|}
\hline & Flow 1 & Flow 2 & Flow 3 & Flow 4 & Flow 5 \\
\hline Measurement & 6 & 5 & 10 & 5 & 7 \\
\hline $\begin{array}{l}\text { "Best" Balance } \\
\text { from MASSBAL }\end{array}$ & 6.05 & 5.04 & 11.09 & 4.69 & 6.40 \\
\hline Error & +0.05 & +0.04 & +1.09 & -0.31 & -0.60 \\
\hline
\end{tabular}

Data reconciliation can be performed with an any system that can solve both a mass/energy balance and an optimization problem. In simple cases, like the examples above, it can be done with commercially available spreadsheet programs. For more complex industrial cases, a process simulation system is required. All the major pulp and paper simulators have at least optional optimizers available, so data reconciliation is possible with any of them. SACDA's MASSBAL II, which has data reconciliation capability built into the standard release of the product, was used for this project.

\section{Implementation of MASSBAL II Model at Bowater}

The MASSBAL II model of the TMP mill with the data reconciliation feature and the interfaces to the PI system were installed at the Bowater Catawba mill. The model was run hourly at the mill. The input data was obtained from the PI database, the model was run and the reconciled data was written back to the PI database. 
The model used 130 process measurements obtained directly from the PI database. When the MASSBAL executive routine is started, these process values are requested from PI by its PI tagname which ends with .PV. These process values are substituted for the default values in the input file of the MASSBAL model. When the model converges, and the reconciled values are obtained, the reconciled values are posted back to the PI data base with a tag ending in .CV. The differences between the process variable and the reconciled value is also written to the PI database and has a tag ending with .DF. For example, 61FC8042.PV is the tag for the measured flow from the line 4 latency chest, 61FC8042.CV is the tag for the reconciled value, and 61FC8042.DF is the tag for the difference between 61FC8042.CV and 61FC8042.PV.

In order to search the data and look for potential problems in the mill, two types of summaries were developed. First, a series of summary displays were created using the standard PI graphical interface. Second a text based exceptions report program was written to scan the MASSBAL output for mismatches between the observed and calculated numbers.

A series of displays were created so that the calculated value from the MASSBAL model can be compared to the actual process variable. These displays were created using the PI system. A time series plot showing both the calculated value and the process value on the same axes was produced along with another graph showing the difference of these two values. From these graphs, one can easily see any discrepancies between the actual and the calculated value. Figure 4 shows an example of the graphical output of results from the model. In Figure 4, both the measured value and the calculated value are shown as a function of time. Figure 5 shows an example of a graphical output for multiple variables. In Figures 4 and 5, information regarding the specific variable has been removed, and these figures are only shown to illustrate the type of information that can be obtained.

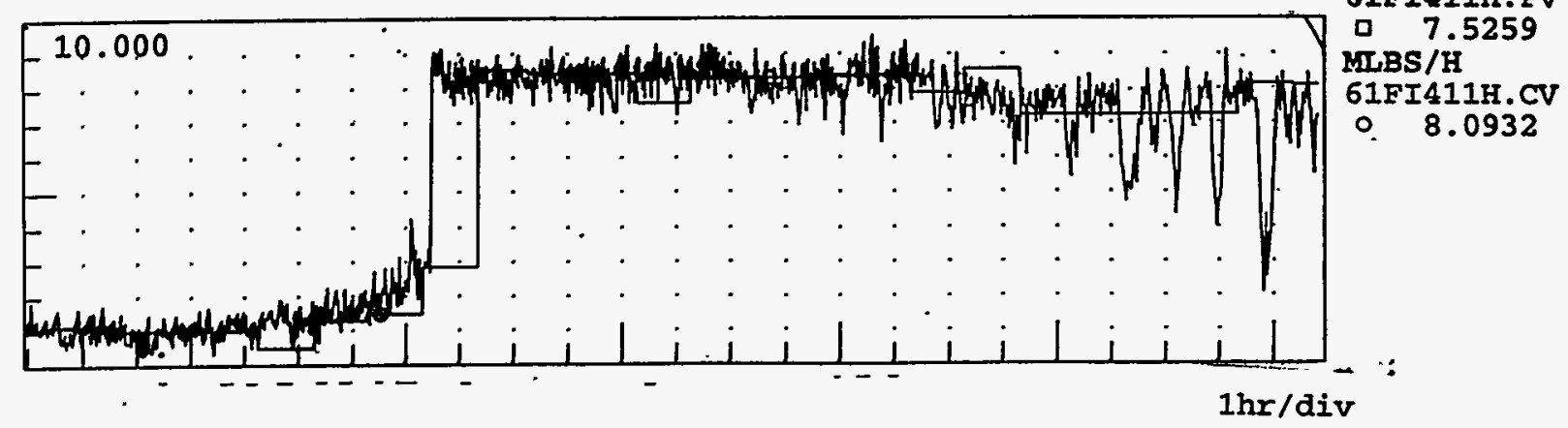

Figure 4. Example of the Graphical Output of MASSBAL Results. 


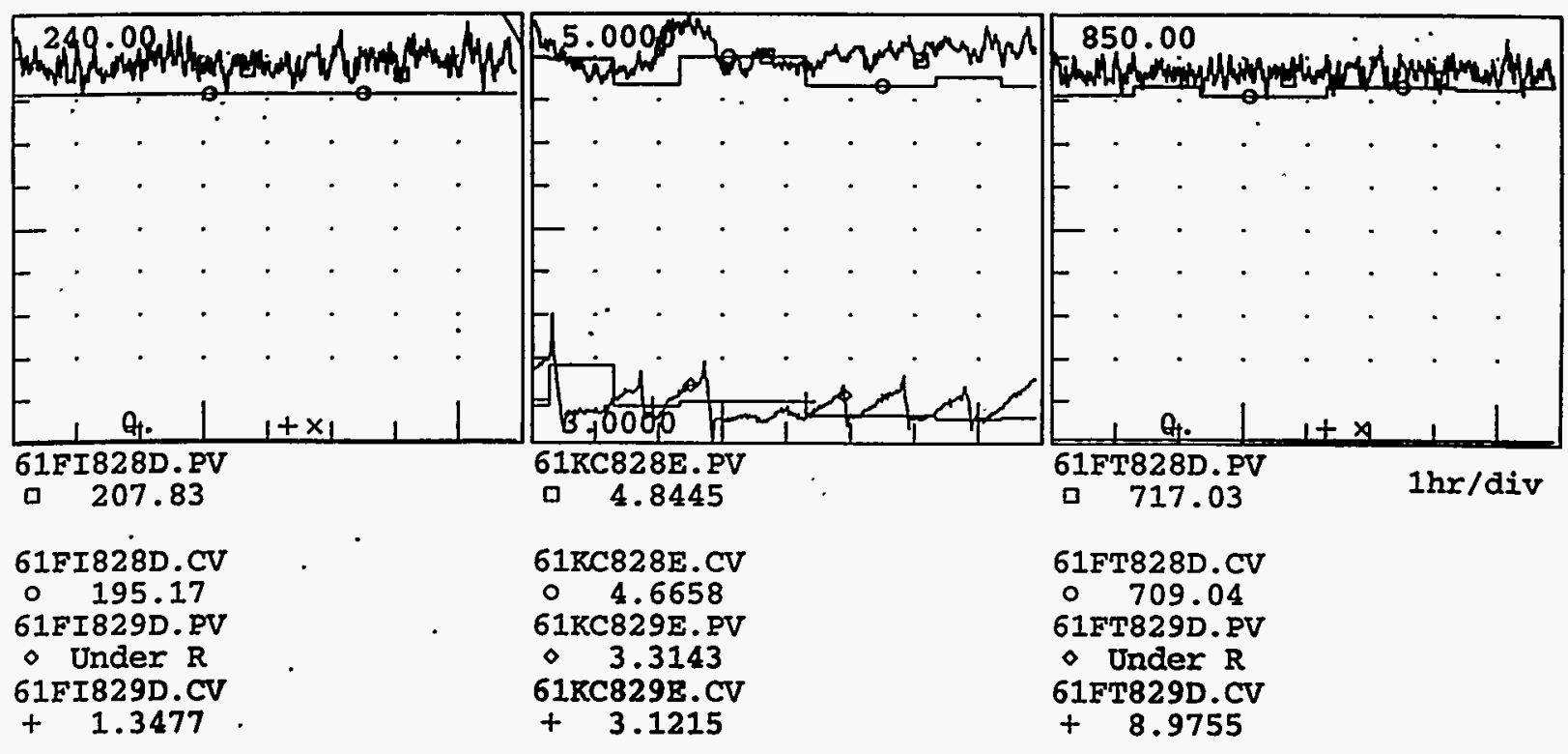

Figure 5. Examples of Multiple Graphical Output of MASSBAL Results.

A daily exceptions report was also developed which summarizes only those values where the model and the process value differ by a specified amount. This report lists the PI tagnames, a description of the measurement, and the process and calculated data. This report was then used to identify for possible problems in the process.

The results of this model were used to indicate possible sensor problems in the mill. Because the MASSBAL model did not predict the quality of the pulp, it did not have high utility to Bowater for the purpose of increasing efficiency, but was helpful for process engineering. Some anomalies in the primary screen dilution flows were uncovered using the reconciled data. The model could be reconfigured as a soft sensor for estimation of critical variables such as chip temperature moisture and density. In order to account for the quality of the pulp, the focus of the project turned to using Process Insights ${ }^{\mathrm{TM}}$ to develop a model of the TMP process. 


\section{PROCESS INSIGHTS ${ }^{\mathrm{TM}}$ MODEL FOR TMP MILL}

Once it was realized that the combined MASSBAL and MAPPS model would not be developed, it was decided to model the TMP mill using a different approach. Process Insights $^{\mathrm{TM}}$ Software of Pavilion Technologies was chosen. Process Insights ${ }^{\mathrm{TM}}$ uses a neural network approach to model a process. The model is built or trained using historical data files of the input and output parameters. In order to use this technique, an adequate database must exist, and the data must have enough variability so that the correct relationships can be obtained.

Previously, Process Insights ${ }^{\mathrm{TM}}$ was successfully used to predict quality parameters on a papermachine [9]. Neural Nets have previously been applied to the refining process $[10,11]$. In one instance [10], it was found that a neural network-based controller could be used to simulate a self-tuning regulator to control the refining process. A neural network model was recently used to model the refining of wood chips [11]. Three input parameters, production rate, specific energy, and refining consistency were used to predict fiber length, the long-fiber fraction, freeness, and breaking length. A representative model was developed from this data. Note, that in [11] there was no input for the quality or temperature of the chips, which one would expect to play a major role in the quality of the pulp.

It was decided that instead of attempting to model the entire TMP process, only one element of the process would be modeled. It was decided to model the refiners on line number four of the Bowater mill. Developing a model to predict the quality of the pulp being produced on line four was the goal of this modeling effort.

\section{Overview of Process Insights ${ }^{\mathrm{TM}}$}

Process Insights ${ }^{\mathrm{TM}}$ is a software that combines the theory of neural networks, chaotic systems theory, and fuzzy logic, to yield a process modeling tool for predictive-adaptive control of a process [6]. The neural networks are nonlinear regression algorithms that can model high-dimensional systems. Chaotic systems theory is used help develop models for seemingly unpredictable processes. Fuzzy logic is used to give soft constraints on parameters for use in optimization or set-point determination. 
In order to develop a Process Insights ${ }^{\mathrm{TM}}$ model the following steps must be conducted: develop a list of input and output variables, collect historical data for variables, format data, preprocess the data, split data into training set and verification set, train model, verify the accuracy of the model. When an accurate model has been developed, variable sensitivities, process optimization, and what-if studies can be conducted.

Once historical data set has been obtained, the most important step to creating a model is the preprocessing of the data. The data must be formatted so that is conforms to the same time scale. Missing data must be estimated, and bad data eliminated. Data corresponding to sensor malfunctions, calibration spikes, shutdowns, or other erroneous data must be removed. If there are time delays for certain variables they must be set. One should also be aware of redundant variables and eliminate them if possible. Once the historical data is ready it is split into a training set, used to train the model, and a verification set, used to verify that the model is accurate.

With Process Insights ${ }^{\mathrm{TM}}$, the training of the model is all completed by the computer. Training is completed when the relative error of the test data is minimized. A completed model is obtained if the relative error of the training data and test data are both small and similar, and the model predicts the output values accurately. If a model with low relative error can not be achieved, the historical data must be looked at closer and missing information added.

Once a trained model has been obtained, it can be used to understand the process. The model can be retrained at any time with new data sets so that it can adapt to changes in the process. Once a model is developed, trained and retained on a regular basis with reliable results it can be used for closed loop control of the process. It is important to note emphasize that the neural net model is based solely on process data and a trained model may not have learned the correct relationship between the input and output variables. This may become evident when a set of conditions that does not correspond to data used in the training set results gives incorrect predictions. A model is only as good as the data that was used to develop the model.

\section{Model Variables}

Eleven input parameters and ten output parameters were chosen to build a Process Insights ${ }^{\mathrm{TM}}$ model. The process parameter data set was complied at Bowater and reflected a 
years worth of data. The input parameter data set, listed in Table 5, was compiled from the PI system historian, and the data interval was five minutes. The quality data set, listed in Table 6, was compiled from an INGRES database resident on the mill VAX system. The quality data was recorded once per shift and was obtained from laboratory tests of pulp and handsheet samples: During each shift, samples of pulp are periodically collected and combined in one pail. Once per shift, the composite sample of pulp is tested to provide the shift average for the quality parameters.

Table 5. Input Parameters for Process Insights ${ }^{\mathrm{TM}}$ Model.

\begin{tabular}{|l|l|l|}
\hline Description & Units & Variable code \\
\hline \hline Clock Time & hrs:min & \\
\hline Primary Refiner \#4 Plate Operating Hours & hrs & PRHR \\
\hline Secondary Refiner \#4 Plate Operating Hours & hrs & SCHR \\
\hline Primary Refiner \#4 Dilution Flow & GPM & PRDL \\
\hline Secondary Refiner \#4 Dilution Flow & GPM & SCDL \\
\hline Primary Refiner \#4 Meter Load & MW & PRLD \\
\hline Secondary Refiner \#4 Meter Load & MW & SCLD \\
\hline Primary Refiner \#4 Plate Position & mil & PMGP \\
\hline Secondary Refiner \#4 Plate Position & mil & SCGP \\
\hline Line \#4 Specific Power & HPD/AD & SPPW \\
& Ton & \\
\hline \#4 Latency Chest Canadian Standard Freeness & ml & CSF \\
\hline \#4 Latency Chest Consistency & $\%$ & CS \\
\hline \#4 Latency Chest Outflow & (GPM) & FLRT \\
\hline
\end{tabular}


Table 6. Output Parameters for Process Insights ${ }^{\mathrm{TM}}$ Model.

\begin{tabular}{|l|l|l|}
\hline Description & Units & Variable code \\
\hline \hline Bauer-McNett Fiber Fraction -100Mesh & $\%$ & BM100 \\
\hline Bauer-McNett Fiber Fraction -200Mesh & $\%$ & BM200 \\
\hline Bauer-McNett Fiber Fraction -28Mesh & $\%$ & BM28 \\
\hline Bauer-McNett fiber Fraction -44Mesh & $\%$ & BM44 \\
\hline Breaking Length & Km & BRLN \\
\hline Bulk & & BULK \\
\hline Burst Factor & & BRST \\
\hline Fines & $\%$ & FINS \\
\hline Shives & $\%$ & SHVS \\
\hline Tear Factor & & TEAR \\
\hline
\end{tabular}

Note, that in Table 5 freeness was listed as an input parameter, but is really a product of the refining, and should be listed as an output variable. Bowater included this in the input parameter list because they have an on-line freeness tester and the data is taken from the PI system database. The specific power is a calculated variable from the other input parameters and was not used in the analysis.

A second set of input parameters were collected at Bowater and are listed in Table 7. These parameters were not included in the initial Process Insights ${ }^{\mathrm{TM}}$ model.

Table 7. Additional Input Parameters Compiled by Bowater.

\begin{tabular}{|l|l|}
\hline Description & Units \\
\hline \hline Chip Surge Bin A Level & $\mathrm{ft}$ \\
\hline Metering Feeder A & $\mathrm{rpm}$ \\
\hline \# 4 PR Cross Screw Speed & $\mathrm{rpm}$ \\
\hline Secondary Refiner \#4 Transfer Conveyor Speed & $\mathrm{rpm}$ \\
\hline \#4 Latency Chest Level & $\mathrm{ft}$ \\
\hline Primary Screen Headbox Temperature & ${ }^{\circ} \mathrm{F}$ \\
\hline
\end{tabular}




\section{Results}

The data sets containing a approximately one year worth of data for the parameters listed in Tables 5 and 6 were transferred to Pavilion Technologies for the purpose of building a model.

Using the Process Insights ${ }^{\mathrm{TM}}$ preprocessor, the input data set was transformed to provide daily averages, then a subset of the data was chosen to create a training set. This set of data was then used to develop a preliminary process model for tear, breaking length, and burst. The resulting Process Insights ${ }^{\mathrm{TM}}$ model could obtain only an $\mathrm{R}^{2}$ value of 0.3 . This result implied that important input information was missing. It is likely that without information on the chip quality, moisture content, and temperature history a good model to predict the quality of the pulp will not be achieved. In addition, Pavilion Technologies advised against using shift averages from the composite pulp samples because the pertinent variation in the data will be averaged out of the final data set.

In order to have obtained the necessary quality data, an extensive testing program would have been required. Several months of data would need to be collected. In addition to the quality data, input parameters such as chip quality, moisture content, and temperature history would need to be collected, which at the time were not available at Bowater. Even with this new data, a successful model may not have been realized. This information in combination with the project history lead to the decision to shut-down the project.

It is important to note that to use Process Insights ${ }^{\mathrm{TM}}$ successfully, one must have adequate historical data. The data must contain enough information to describe the model adequately. Although it appears to be common sense, it can not be stressed enough that if a parameter of fundamental importance is not represented in some manner, a good model can not be developed.

\section{Analysis of Results}

In order to understand why the data set compiled by Bowater was not adequate to develop a Process Insights ${ }^{\mathrm{TM}}$ model and provide some details to help with future attempts in modeling TMP refining, the data was analyzed with more traditional methods. 
The statistics of both the shift average input and output data sets were computed. A summary of the results is given in Table 8. In general, the variation in the input parameters is greater than the output parameters with percentage shives being the exception.

Table 8. Summary of Statistics for Process Insights ${ }^{\mathrm{TM}}$ Variables.

\begin{tabular}{|l|l|l|l|l|}
\hline Variable & $\begin{array}{l}\text { Number of } \\
\text { points }\end{array}$ & Mean & $\begin{array}{l}\text { Coeff. of } \\
\text { Variation }\end{array}$ & $\begin{array}{l}\text { Standardized } \\
\text { Skewness }\end{array}$ \\
\hline PRHR & 929 & 295.7 & 65.5 & 4.6 \\
\hline SCHR & 922 & 289.7 & 66.6 & 5.9 \\
\hline PRDL & 761 & 43.5 & 21.6 & -4.4 \\
\hline SCDL & 782 & 38.2 & 23.1 & -36.9 \\
\hline PRLD & 782 & 7.70 & 22.1 & -43.2 \\
\hline SCLD & 782 & 6.97 & 22.1 & -43.6 \\
\hline PMGP & 863 & 251.38 & 26.6 & -1.9 \\
\hline SCGP & 860 & 264.8 & 28.1 & -6.8 \\
\hline CSF & 878 & 132.6 & 17.6 & 1.5 \\
\hline CS & 939 & 3.79 & 19.0 & -12.9 \\
\hline FLRT & 801 & 758.8 & 20.4 & -46.8 \\
\hline BM100 & 994 & 11.23 & 14.7 & 18.0 \\
\hline BM200 & 997 & 6.91 & 17.5 & 13.0 \\
\hline BM28 & 997 & 28.70 & 15.1 & -8.9 \\
\hline BM44 & 997 & 20.70 & 7.6 & 5.6 \\
\hline BRLN & 997 & 2289 & 9.2 & 1.8 \\
\hline BULK & 997 & 3.16 & 6.2 & 6.4 \\
\hline BRST & 997 & 12.08 & 11.2 & 1.8 \\
\hline FINS & 997 & 33.4 & 9.5 & 14.1 \\
\hline SHVS & 997 & 1.07 & 55.7 & 16.4 \\
\hline TEAR & 997 & 67.45 & 10.2 & 2.2 \\
\hline & & & & \\
\hline
\end{tabular}

A cross correlation analysis of the shift averaged data-set was completed. Table 9 provides the cross correlation coefficients for the input parameters, Table 10 provides the cross correlation coefficients of the output parameters, and Table 11 provides the correlation coefficients for the input parameters compared to the output parameters. For this analysis, 735 data sets were used, and where the confidence level of the coefficient 
was less than $95 \%$, the number is shown with italics. Note, that only low correlation coefficients have low significance.

Table 9. Cross Correlation Coefficients for Input Parameters.

Correlation Coefficient, Symmetric Matrix

Italicized coefficients have a confidence level of less than 95\%

\begin{tabular}{|l|l|l|l|l|l|l|l|l|l|l|}
\hline & SCHR & PRDL & SCDL & PRLD & SCLD & PRGP & SCGP & CSF & CS & FLRT \\
\hline PRHR & .6985 & .0308 & -.0260 & 0.0624 & -.0298 & .5236 & .3964 & -.0931 & .2166 & .1053 \\
\hline SCHR & 1.000 & .0301 & .0076 & .0631 & -.0434 & .3553 & .5012 & -.1122 & .1992 & -.0920 \\
\hline PRDL & & 1.000 & .0888 & .3923 & .4145 & .2677 & .0133 & .1278 & .2524 & .3261 \\
\hline SCDL & & & 1.000 & .5322 & .5030 & .0996 & -.0063 & -.1057 & -.1014 & .5700 \\
\hline PRLD & & & & 1.000 & .6644 & .1992 & .1790 & -.0877 & .3100 & .5734 \\
\hline SCLD & & & & & 1.000 & .0974 & .0499 & -.0358 & .2398 & .5898 \\
\hline PMGP & & & & & & 1.000 & .3064 & -.1331 & -.0041 & .2114 \\
\hline SCGP & & & & & & & 1.000 & -.0029 & .1113 & .1730 \\
\hline CSF & & & & & & & & 1.000 & .2452 & -.0209 \\
\hline CS & & & & & & & & & 1.000 & -.0732 \\
\hline FLRT & & & & & & & & & & 1.000 \\
\hline
\end{tabular}

Table 10. Cross Correlation Coefficients for Output Parameters.

Correlation Coefficient, Symmetric Matrix

Italicized coefficients have a confidence level of less than $95 \%$

\begin{tabular}{|l|l|l|l|l|l|l|l|l|l|l|}
\hline & BM100 & BM200 & BM28 & BM44 & BRLN & BULK & BRST & FINS & SHVS & TEAR \\
\hline BM100 & 1.000 & .5970 & -.4556 & .3734 & .0454 & -.4325 & .0085 & -.1560 & -.2540 & -.3579 \\
\hline BM200 & & 1.000 & -.2350 & .2583 & 0.000 & -.4246 & -.1827 & -.0950 & -.0806 & -.4830 \\
\hline BM28 & & & 1.000 & -.5009 & -.2014 & .4426 & -.1758 & -.5962 & .5080 & .3164 \\
\hline BM44 & & & & 1.000 & .1440 & -.3559 & .0610 & -.1200 & -.3153 & -.2661 \\
\hline BRLN & & & & & 1.000 & -.5826 & .7500 & .1683 & -.2533 & .1854 \\
\hline BULK & & & & & & 1.000 & -.4614 & -.1629 & .3549 & .1875 \\
\hline BRST & & & & & & & 1.000 & .1401 & -.3186 & .4648 \\
\hline FINS & & & & & & & & 1.000 & -.2435 & -.0517 \\
\hline SHVS & & & & & & & & & 1.000 & .0333 \\
\hline TEAR & & & & & & & & & & 1.000 \\
\hline
\end{tabular}


Table 11. Cross Correlation Coefficients for Input versus Output Parameters.

\section{Correlation Coefficient}

Italicized coefficients have a confidence level of less than $95 \%$

\begin{tabular}{|l|c|c|c|c|c|c|c|c|c|c|}
\hline & BM100 & BM200 & BM28 & BM44 & BRLN & BULK & BRST & FINS & SHVS & TEAR \\
\hline PRHR & .2660 & .2234 & -.2738 & .2524 & .1296 & -.2171 & .0552 & .1015 & -.1073 & -.1469 \\
\hline SCHR & .2446 & .2958 & -.2174 & .1878 & .0821 & -.2827 & .0266 & .0788 & -.1505 & -.1258 \\
\hline PRDL & -.3069 & -.0374 & .2990 & -.1727 & .0035 & .1006 & -.0202 & -.0638 & .3699 & .1184 \\
\hline SCDL & -.0230 & .0187 & -.0920 & -.0467 & .1200 & -.0435 & .0951 & .1059 & -.1117 & -.0514 \\
\hline PRLD & -.0182 & .0004 & .0274 & -.0797 & .1259 & -.0849 & .1481 & .0670 & .0138 & .1507 \\
\hline SCLD & -.1577 & -.0302 & .1211 & -.0868 & .0065 & .0750 & .0047 & -.0097 & .0022 & .0605 \\
\hline PMGP & .1681 & .2424 & -.2330 & .2536 & .2657 & -.2923 & .1656 & .1143 & -.2266 & -.1288 \\
\hline SCGP & .2193 & .2343 & -.2417 & .1944 & .1250 & -.3158 & .1273 & .1322 & -.1917 & -.0861 \\
\hline CSF & -.1868 & -.0264 & .2067 & -.0073 & -.0441 & .1281 & -.1263 & -.0347 & .2069 & .0425 \\
\hline CS & .0372 & .1806 & .2210 & -.0905 & -.1334 & .0561 & -.2150 & -.0496 & .3360 & .0375 \\
\hline FLRT & -.1371 & -.0961 & -.0626 & .0219 & .0574 & -.0243 & .1003 & .0646 & -.1435 & .0163 \\
\hline
\end{tabular}

The cross correlation components given in Table 9 show that many of the input parameters are highly correlated. For instance, the primary and secondary refiner plate lives have a correlation coefficient of 0.6985 , indicating that they are similar data sets. This could be expected since the plates on the primary and secondary refiners probably will be changed at the same time when the line is down. In addition, the plate age and plate gap are highly correlated, because as the plate ages, the gap decreases to account for wear. The meter loads on the primary and secondary loads appear to be highly correlated to the dilution flows, and all these values are correlated to the latency chest outflows. This high correlation of the input variables indicates that the variables are not truly independent of one another.

When the correlation coefficients of the output matrix were studied, it was found that breaking length and burst were highly correlated to one another. In addition, burst and tear appear to be somewhat correlated. This correlation could be expected because as the quality of the pulp increases the strength characteristics increase.

Table 11 shows that there are no strong correlation's between the input parameters and the output parameters. This suggests that the relationship is dependent on an interaction of the 
input parameters or that the critical parameters are missing from the input data. The low $\mathrm{R}^{2}$ value of the Process Insights ${ }^{\mathrm{TM}}$ model suggests that critical information is missing.

For a comparison to the Process Insights ${ }^{\mathrm{TM}}$ model, multiple linear regression models for the 10 output parameters and the freeness were developed. The results of this model are given in Table 11. None of the models could explain more than thirty percent of the variation in the data. This result is in agreement with the Process Insights ${ }^{\mathrm{TM}}$ model. The best fit was for shives, where the percentage of shives was most influenced by the primary dilution followed by the primary gap opening.

Table 11. Results from Linear Regression.

\begin{tabular}{|l|l|l|l|l|}
\hline Variable &. $\mathrm{R}^{2}$ & & Variable & $\mathrm{R}^{2}$ \\
\hline BM100 & 0.19 & & CSF & 0.15 \\
\hline BM200 & 0.12 & & BRLN & 0.11 \\
\hline BM28 & 0.26 \\
\hline BM44 & 0.14 \\
\hline FINS & 0.03 \\
\hline SHVS & 0.36 & & BULK & 0.18 \\
\hline & & BRST & 0.12 \\
\hline & & TEAR & .08 \\
\hline
\end{tabular}

It is concluded that the data set compiled for the Process Insights ${ }^{\mathrm{TM}}$ model is missing some important parameters. Likely candidates are chip temperature, moisture, and density. 


\section{CONCLUSIONS AND RECOMMENDATIONS}

Controlling the pulping process with the use of a process model is probably, the correct approach to be taken in the paper industry. If a process can be optimized, energy and raw material consumption can be minimized while quality is maintained. The development of such a model for a thermomechanical pulp mill was the objective of the present project. Although this objective was not met in the present work, it appears to be an obtainable goal; especially as advances in on-line sensors and process modeling are made.

The initial goal of combining the process modeling software of MASSBAL and MAPPS was not a success, and the project was never able to fully recover from this setback. A MASSBAL model of the process was developed, but had limited use since it did not predict the quality of the pulp. At that point, instead of developing new models based on first principles, a neural network model was investigated. It was found that the process data available for training was not sufficient to develop a model with accurate predictions of pulp quality. With this knowledge, it was decided to shut the project down instead of embarking on a new approach or beginning an extensive data collection program.

By reviewing the actions and results of this project, a better program approach can be developed. In order to develop a working model, one must first establish the physics of the process. Second, one must establish how the physical characteristics are generated in the pulp and what process parameters affect these properties. Third, one must be able to measure the important process and quality parameters. Finally, one must have an adequate method to develop a predictive model. Once the accuracy of the model has been established, it can be used to aid in the control of the process.

The quality parameters to be predicted; such as tear, breaking length, bulk, freeness, and fiber fraction; must be established. It is essential to determine which properties of the pulp affect the parameters of interest. For instance, breaking length is affected by fiber strength, fiber length, relative bonded area, intrinsic bonding strength, and degree of fiber curl. This is where paper physics can play an important role in establishing these relationships and providing theoretical or predictive equations. Much of this type of information has already been developed and can be obtained from the literature.

The pulp characteristics will be affected by the fiber source and the process. It is important to understand how the process changes the characteristics of the pulp. For example, 
increasing refining level increases fiber conformability, which in turn increases the relative bonded area, and an increase in strength may be realized. On the other hand, increased refining could decrease fiber length and the strength may decrease. Refining at high consistency may increase fiber curl and produce poor strength. In addition, refining at higher temperatures creates a more conformable fiber without inducing excessive fiber damage; thus one gets a stronger sheet. This is one of the driving reasons that lead to the development of TMP. With identification of the important pulp characteristics and the process parameters that alter these characteristics, a reasonable list of the process parameters that need to be monitored can be written down.

Once a list of essential parameters is prepared, it must be determined if they can and are being measured. This is the key to controlling a well optimized process. Without the proper sensors and instrumentation being collected, a model will be useless. This is where the current project fell short. The need for on-line sensors was never stressed as an important part of the research program. It was assumed that Bowater had all the necessary data that was needed to predict the quality of the pulp. Of course this was not true, and probably not true of most pulp mills. Fiber source or chip quality probably have a large impact on pulp quality and this information was not available. If the information is not available and it is expected to impact the output parameters, than no model will provide an accurate solution!

Once it is established that the necessary parameters can be measured, a model must be developed. An approach such as that used by Process Insights ${ }^{\mathrm{TM}}$ can lead to an accurate model that can be used for control. Because the model learns from actual process history, it can uncover the complex nonlinear relationships between the input parameters which produce the output parameters. Of course this depends on the availability of the necessary data. In addition, the historical data must contain process conditions similar to that being predicted. As a process changes over time, the model can be updated. If certain data is unavailable or can not be measured, first principle models can be used to predict the missing data. Of course, models of these types are limited by modeling assumptions and the accuracy of the input parameters. No matter what the modeling technique invoked, detailed knowledge of the process must be known and accounted for.

Once the accuracy of a model has been established, it can be used to control the process with the ultimate goal of closed loop control. Today's computer technology with the modeling capabilities available probably make it possible to develop closed loop control. 
The industry probably lacks in the same areas where this project lacked. That is a lack of proper process data and hence sensors which provide essential information on the physical state of wood chips and pulp.

\section{REFERENCES}

1. Institute of Paper Science and Technology, Development and Demonstration of the Use of Modular Thermomechanical Pulpmill Simulation Models to Develop Energy Reduction Strategies: Report 1, DOE Contract DE-GF02-90CE40937, Prepared for Keith Adkins, Contracts Specialist, Argonne, Illinois, August 15, 1991.

2. MAPPS, User's Guide, The Institute of Paper Science and Technology, Atlanta, Georgia, 1991.

3. MASSBAL 2.0. Process Simulation Software, SACDA, Inc., 343 Dundas Street, Ste. 500 , London, Ontario Canada, N6b 1V5.

4. Dodds, G. IPST/DOE TMP Modeling Project at Bowater: Model Documentation, Revision 0, SACDA, Inc. Ontario, Canada, August 1994. pp. 30.

5. Jonason, N. B., Development, Validation and Sensitivity Study of a TMP Pulp Mill, A190 Research Report, Institute of Paper Science and Technology, March 9, 1991.

6. Process Insights ${ }^{\mathrm{TM}}$ User's Guide, Version 2.0, Pavilion Technologies Incorporated, Austin Texas, 1993.

7. Ham, P. G., Cleves, G. W., and Lawler, J. K., "Operating Data Reconciliation: An Aid to Improve Plant Performance," Proceedings of the National Petroleum Refiners Association Computer Conference, CC85, October, 1985.

8. Wells, H. A., Fundamentals of Process Control for the Pulp and Paper Industry, Edited N. Snell, Tappi Press, Atlanta, GA, 1995, pp. 500.

9. Schweiger, C. A., and Rudd, J. B., "Prediction and Control of Paper Machine Parameters Using Adaptive Technologies in Process Modeling," Proceedings of the 1994 TAPPI Process Control Symposium.

10. Kooi, S. B. and Khoransanim K., "Control of the Woodchip Refiner Using Neural Networks," Tappi Journal, Vol. 75, No. 6, 1992, pp. 156-162.

11. Y. Qian, P. Tessier, and G. A. Dumont, "Modeling a Wood-chip Refiner Using Artificial Neural Networks," Tappi Journal, Vol. 78, No. 6, 1995, pp. 167-174.

\section{ACKNOWLEDGMENTS}

The financial and administrative support of the Office of Industrial Technology of the United States Department of Energy is gratefully acknowledged. The participation and support of Bowater Incorporated especially Mr. Benjy James and Ms. Jennifer Terrel are gratefully recognized. 